\title{
Changes in volatile composition of Madeira wines during their oxidative ageing
}

\author{
J.S. Câmara ${ }^{\mathrm{a}, *}$, M.A. Alves ${ }^{\mathrm{b}}$, J.C. Marques ${ }^{\mathrm{a}}$ \\ ${ }^{a}$ Madeira Chemistry Research Center, Depto. de Química da Universidade da Madeira, Campus Universitário da Penteada, $9000-390$ Funchal, Portugal \\ ${ }^{\mathrm{b}}$ Depto. de Eng ${ }^{a}$ Química, Faculdade de Engenharia da Universidade do Porto, Rua Roberto Frias, 4099 Porto, Portugal
}

Received 4 August 2005; received in revised form 12 October 2005; accepted 18 October 2005

Available online 23 November 2005

\begin{abstract}
The influence of the age in the volatile composition of Madeira wines made with Boal, Malvazia, Sercial and Verdelho varieties and aged in oak barrel during 1, 11 and 25 years old was been studied. For this purpose, the evolution of volatile compounds: higher alcohols, ethyl esters, fatty acids, furan compounds, enolic compounds, $\gamma$-lactones, dioxanes and dioxolanes, of the four most utilised varieties were determined using liquid-liquid extraction with dichloromeihane. Octan-3-ol was used as internal standard. The wines made with these varieties showed great differences in sugar content and small variations on $\mathrm{pH}$ and alcoholic degree.

The results show that during ageing, the concentration of fatty acids ethyl esters, acetates and fatty acids decrease significantly contrarily to the great increase of ethyl esters of diprotic acids. There is a strong correlation between sotolon, 2-furfural, 5-methyl-2-furfural, 5-hydroxymethyl2-furfural and 5-ethoxymethyl-2-furfural with wine ageing. These findings indicate that these compounds can be used as ageing wine markers. Among the molecules studied, sotolon [3-hydroxy-4,5-dimethyl-2 $(5 \mathrm{H})$-furanone] was one of the few molecules present in concentrations above the perception threshold in Madeira wines. 5-Eihoxymethyl-2-furfural formed from 5-hydroxymethyl-2-furfural and 2-furfural, derived from sugars, are also involved in the aroma of sweet fortified white wines aged in oxidative conditions. The sensory properties change significantly after long periods of conservation.
\end{abstract}

(C) 2005 Elsevier B.V. All rights reserved.

Keywords: Volatile compounds; Wine aroma; Ageing; Oxidation; Madeira wines

\section{Introduction}

Aroma is an important factor in quality control and quality assurance of foods, but in wines, this factor is possibly the most important. It is produced by a complex balance of more than 800 volatile compounds in different ranges of concentrations, and with different volatilities and polarities. These compounds have differentiated origins - from grapes (varietal aroma), from alcoholic fermentation under anaerobic conditions (fermentative aroma) and the bouquet, which results from the transformation of the aroma during ageing.

During ageing the wine acquires aromatic complexity as a result of important modifications consequence of different phenomena, such as: esterification/hydrolysis reactions, redox reactions, spontaneous clarification, $\mathrm{CO}_{2}$ elimination, slow and

\footnotetext{
* Corresponding author. Tel.: +351 291705112; fax: +351 291705149.

E-mail address: jsc@ uma.pt (J.S. Câmara).
}

continuous diffusion of oxygen through wood pores and transference of tannins and aromatic substances from wood into the wine. The aroma formed during the alcoholic fermentation decrease, but new compounds appear from oak wood and from the evolution of the primary and secondary aromas.

The volatile compounds extracted from the wood, which depends on the quantity of compounds that are potentially extractable, and contact time between wine and wood, have distinct impacts on the aroma of the wine. Thus, among the aromatic aldehydes, vanillin can have a great influence on the aroma of the wine, while 2-furfural and 5-methyl-2-furfural have a small influence. However, 2-furfural would seem to modify the perception of the oak lactones of the wine.

The vinification and ageing processes used for Madeira wines are unique. The fermentation process is stopped by the addition of natural grape spirit in order to obtain an ethanol content of $18-19 \%(\mathrm{v} / \mathrm{v})$. The new wine is then placed in large coated vats and the temperature is slowly increased - at about $5{ }^{\circ} \mathrm{C}$ per day - and maintained at $45-50^{\circ} \mathrm{C}$ for 3 months. After this treatment, 
the wine is allowed to undergo a normal maturation process in oak casks for a minimum of 3 years. The noble varieties from which Madeira wine is produced are Boal, Malvazia, Sercial and Verdelho. The varietal characterisation of these varieties was studied by the first time by Câmara et al. [1]. Due to their alcohol content (16-18\%), yeast is not involved in the ageing process of Madeira fortified wines.

Madeira wines suffers long periods of ageing, 20 years at minimum, in cellars which temperatures at storage conditions arising $30-35^{\circ} \mathrm{C}$ by sun influence, and a humidity degree higher than $70 \%$. The thermal treatment generates thermo-degradation of some compounds that produces numerous volatile and aromatic compounds (furanic aldehydes, volatile phenols, phenolic aldehydes and phenyl ketones). Thus, the changes in chemical composition and consequently on the sensorial perception of Madeira wine during conservation, can be related primarily to the temperature that the wine is stored and with humidity level. The positive effects conferred by oak barrels that include stability and spontaneous clarification, make their use very common.

Traditionally are aged in oak barrels of various sizes $(225$, 640 and 1500-50001) to facilitate the diffusion of oxygen. This is assumed to play a major role in the many chemical reactions occurring during ageing. The presence of the oxygen enhances oxidation reactions in which the anthocyanins, flavonoids and tartaric esters of hydrocynamic acids (phenolic compound of the wine) as well as the phenolic compounds extract from the wood are involved. Oxidation results in organoleptic modifications, generally described as "oxidised" in dry wines, "rancio" in sweet fortified red wines and "maderized" in sweet fortified white wines [2]. On the other hand, oxidation phenomena are generally considered to be favourable, or even indispensable, for the proper development of the aroma of sweet fortified wines.

Cantarelli [3] showed the importance of the carbonyl group of aldehydes as well as the role of amino acids in the formation of the "rancid" odour in maderized wines. In the case of dry wines, these phenomena lead to the deterioration of the bouquet during ageing.

The aim of this investigation was to evaluate the evolution of volatile composition of Madeira wines from the four most representative varieties made with Boal, Malvazia, Sercial and Verdelho after 1, 11 and 25 years of ageing, and enables a comparative study of the wines obtained after different lengths of time. We try to show what happens to Madeira wine aroma after different storage periods. The barrels remained in the winery under the same conditions of temperature and humidity. The evolution of higher alcohols, fatty acids ethyl esters, fatty acids, furanic compounds, namely 2-furfural, 5-methyl-2-furfural and 5-hydroxymethyl-2-furfural, enolic compounds, $\gamma$-lactones and acetals, during ageing was also studied.

\section{Material and methods}

\subsection{Wine samples}

The 86 samples of Madeira wine used in this study were made from 4 different varieties that corresponding different types: Malvazia (sweet) 22 samples-8 with 1 year, 8 with 11 years and 6 with 25 years; Boal (medium sweet) 26 samples-10 with 1 year, 8 with 11 years and 8 with 25 years; Verdelho (medium dry) 21 samples -8 with 1 year, 8 with 11 years and 5 with 25 years; Sercial (dry) 17 samples-7 with 1 year, 6 with 11 years and 4 with 25 year old. All wines were matured in oak barrels. These samples were supplied by Instituto do Vinho da Madeira (40 samples) and Madeira Wine Company (46 samples) and correspond to generic wines submitted to the traditional heating process. Non-heated young wines were also included in order to evaluate any possible influence.

The wines were made following the standard traditional winemaking procedures used for Madeira wine. The grapes are destemmed, crushed and $50 \mathrm{mg}^{-1} \mathrm{SO}_{2}$ was added. Fermentation process is stopped by the addition of alcohol in order to obtain an ethanol content of $18-19 \%(\mathrm{v} / \mathrm{v})$, and took place in oak barrels without yeast inoculation. All samples were matured in oak barrels, submitted to the same oxidative storage conditions. After sampling were stored at $-28^{\circ} \mathrm{C}$ until analysis.

\subsection{Analytical methods}

\subsubsection{Classical enological parameters}

The most usual enological parameters were determined according to Office Internatioi Vigne et Vin (OIV) methods (ethanol, \%, v/v; total and free $\mathrm{SO}_{2} ; \mathrm{pH}$ ), and volatile and titrable acidity according to the methods given by European Economic Community (EEC) (1990).

Total sugar, reducing sugars, dry extract, density, Baumé degree, ash and ash alkalinity were determined according to the methods described in the "Portuguese Official Standards (NP) for Spirits and Alcoholic Beverages": NP 2224, 1988; NP 2223, 1988; NP 2222, 1988; NP 2142, 1986; NP 2221, 1986; NP 2279, 1988 , respectively. All these parameters were measured in triplicate. They are directly correlated with wine quality and stability, and some of them, such as $\mathrm{pH}$ and ethanol degree have a direct influence on ethanolysis of wood components.

\subsubsection{Volatile compounds extraction}

2.2.2.1. Major compounds-liquid-liquid extraction (LLE) and gas chromatography-flame ionisation detector (GC-FID) analysis. Quantitative analysis of major compounds was carried out according with the "Portuguese Official Standards for Spirits and Alcoholic Beverages" NP 3263, 1990. The extract was then analysed by GC (HP5890 series II gas chromatograph) with FID detection. The column was a BP 20 from SGE, $30 \mathrm{~m} \times 0.32 \mathrm{~mm}$ with a $0.25 \mathrm{~mm}$ film thickness. The carrier gas was $\mathrm{He}$ at $1 \mathrm{ml} \mathrm{min}{ }^{-1}$ and the injection was performed in split mode with a split flow of $12 \mathrm{ml} \mathrm{min}^{-1}$. The temperature program was as follows: $45^{\circ} \mathrm{C}$ for $2 \mathrm{~min}$, increased to $220^{\circ} \mathrm{C}$ at $2{ }^{\circ} \mathrm{C} \mathrm{min}{ }^{-1}$. The injector and detector were kept both at $250^{\circ} \mathrm{C}$. Quantitative data were obtained by interpolation of relative peak areas in the calibration plots built by the analysis of synthetic wines containing known amounts of the analytes. 4-Methylpentan-2-ol was used as internal standard.

2.2.2.2. Minor compounds-LLE and ion trap mass spectrometry (GC/ITMS) analysis. The extraction procedure of volatile 
compounds is in according to the method described by Silva Ferreira et al. [4]. Wine samples were treated as follows: $50 \mathrm{ml}$ of aged samples Madeira wines was spiked with $50 \mu$ lof octan-3-ol in hydro-alcoholic solution $(1: 1, \mathrm{v} / \mathrm{v})$ at $422 \mathrm{mg} \mathrm{l}^{-1}$ as internal standard and $5 \mathrm{~g}$ of anhydrous sodium sulphate (higher ionic strength, increases extractability). The mixture was extracted twice using $5 \mathrm{ml}$ of dichloromethane (Lab Scan) each time. The organic layers obtained were dried with anhydrous sodium sulphate (Merck). Two milliliters of this organic extract was concentrated five-fold, under a nitrogen stream, according to the method proposed for Port wines [5]. Two microliters of the extract was injected into the GC with an MS detector.

\subsubsection{Gas chromatography-mass spectrometry (GC/MS) conditions}

The dichloromeihane extracts from different aged Madeira wines, were analysed by GC/MS using a Varian STAR 3400Cx series II gas chromatograph, equipped with a $30 \mathrm{~m} \times 0.25 \mathrm{~mm}$ i.d., $0.25 \mu \mathrm{m}$ film thickness, Stabilwax fused silica capillary column, connected to a Varian Saturn III mass selective detector, according to the method described by Câmara et al. [6]. The chromatographic conditions were: $\mathrm{He}$ (purity $=99.999 \%$ ) as carrier gas $\left(40 \mathrm{~cm} \mathrm{~s}^{-1}\right)$; injector temperature, $250^{\circ} \mathrm{C}$; temperature of the transfer line, $220^{\circ} \mathrm{C}$. A temperature program was used with an initial oven temperature of $40^{\circ} \mathrm{C}$ for $1 \mathrm{~min}$. The temperature was increased in three steps: $40-120^{\circ} \mathrm{C}$ at $1{ }^{\circ} \mathrm{C} \mathrm{min}-1$; $120-180^{\circ} \mathrm{C}$ at $1.7^{\circ} \mathrm{C} \mathrm{min}^{-1} ; 180-220^{\circ} \mathrm{C}$ at $25^{\circ} \mathrm{C} \mathrm{min}^{-1}$. Each step was preceded by a small period at constant temperature of 2,1 and $10 \mathrm{~min}$, respectively. The analysis of these compounds was carried out with an injection in splitless mode. Detection was performed by Saturn III mass spectrometer in the EI mode (ionisation energy: $70 \mathrm{eV}$; source temperature: $180^{\circ} \mathrm{C}$ ). The acquisition was made in full scan mode (mass range $30-300 \mathrm{~m} / \mathrm{z}$; 1.9 spectra $^{-1}$ ).

The data outlined in the tables correspond to the arithmetic average of the results obtained from the two extractions that were made from each sample. Since the repeatability of the chromotographic method was good only three injections of dichloromethane extracts was carried out.

\subsection{Statistical analysis}

Significant differences among the four Madeira wines varieties for each of the constituents were determined by one-way analysis of variance (ANOVA) using a Statistical Package for Social Sciences (SPSS) Program, Version 11.0 (SPSS Inc., 2003). Principal component analysis (PCA) and stepwise linear discriminant analysis (SLDA) were performed using the same SPSS program. These techniques were applied to the normalised relative amounts of the volatile compounds. PCA is an unsupervised technique that reduces the dimensionality of the original data matrix retaining the maximum amount of variability [7]. SLDA is a supervised method used for classification purposes.

\section{Results and discussion}

\subsection{Enological parameters}

Table 1 shows the enological variables quantified in Madeira wine samples with 1,11 and 25 years old. As can be seen, average $\mathrm{pH}$ values are almost invariant for all samples studied, which lie between 3.41 and $3.69\left(20^{\circ} \mathrm{C}\right)$. The average content of total and fix acidity seems to increase with ageing for all varieties of Madeira wines, particularly for samples with 25 years old. The volatile acidity also increase with wine age namely for dry (Sercial) and medium dry (Verdelho) samples.

For all Madeira wine varieties studied the obtained values for alcoholic degree are among 15 and 22\%, which is in agreement with European Union (EU) regulation for liquor wines (EU No. 4252). Alcoholic degree present average values up to $16 \%$ for younger wines samples, but a small increment above $20 \%$ could be observed for Boal (medium sweet) and Sercial (dry) wines with 25 years old.

As was expected the average content of total sugar increase clearly with time of storage and with the sweetness degree. The total dry extract, density and Baumé degree $\left(20^{\circ} \mathrm{C}\right)$ present a big variation with wine variety, increasing with the sugar content and with wine ageing.

Small differences were observed in the average content of ash and ash alkalinity between varieties and with wine time storage. Concerning the sulphur dioxide, the average levels are clearly below the maximum admissible limits $\left(200 \mathrm{mg} 1^{-1}\right)$ for samples having more than $5 \mathrm{~g}^{-1}$ of total sugar contents (EU No. 4252, 1988).

\subsection{Volatile compounds versus time of storage}

During the ageing period the complexity of wine aroma is increased because the extraction of certain compounds from wood, by example the oak lactones and volatile phenols (smoky aroma) which are transferred to wine [8].

By means of the used procedure over 120 compounds were identified in the aroma volatiles of aged Madeira wines, the majority being higher alcohols (mainly isoamyl alcohols and 2-phenylethanol), ethyl esters of medium-chain acids (hexanoic and octanoic acids) and furanic derivatives. Among the other components present were: $4 C_{13}$ compounds, the isomeric vitispiranes, $\beta$-damescenone and TDN, probably formed by carotenoid degradation present in the grapes; 2 monoterpene oxides-trans-furan linalool oxide and cis-furan lonalool oxide; 18 fatty acids; 8 aldehydes, numerous furan derivatives (20) which concentration increase with time, $6 \gamma$-lactones and the cis-/trans-isomers of whisky lactone, the isomers of dioxanes and dioxolanes and some enolic derivatives. Only two sulphur-containing compounds were identified: methionol and benzothiazol.

Fig. 1 shows the composition of the aroma volatiles of two samples from Malvazia wines with 1 and 25 years old. The initial characteristic aroma of a young sweet fortified white wine subjected to long ageing in the presence of oxygen changed considerably over time. Is significant the evolution of some volatile 
Table 1

Evolution of the enological and physicochemical parameters (mean values, $n=3$ ) of Madeira wines with ageing

\begin{tabular}{|c|c|c|c|c|c|c|c|c|c|c|c|c|}
\hline & \multicolumn{12}{|l|}{ Variety } \\
\hline & \multicolumn{3}{|l|}{ Boal } & \multicolumn{3}{|c|}{ Malvazia } & \multicolumn{3}{|l|}{ Sercial } & \multicolumn{3}{|c|}{ Verdelho } \\
\hline & $1^{\mathrm{a}}$ & $11^{\mathrm{a}}$ & $25^{\mathrm{a}}$ & 1 & 11 & 25 & 1 & 11 & 25 & 1 & 11 & 25 \\
\hline \multicolumn{13}{|l|}{ Composition } \\
\hline \multicolumn{13}{|l|}{ Acidic } \\
\hline $\mathrm{pH}$ & 3.64 & 3.59 & 3.49 & 3.45 & 3.48 & 3.42 & 3.41 & 3.47 & 3.51 & 3.69 & 3.62 & 3.47 \\
\hline Volatile acidity $\left(\mathrm{g}^{-1}\right)$ & 0.19 & 0.53 & 1.27 & 0.25 & 0.67 & 0.81 & 0.44 & 0.76 & 1.29 & 1.34 & 0.73 & 2.36 \\
\hline Fix acidity $\left(\mathrm{g}^{-1}\right)$ & 4.9 & 4.8 & 6.3 & 6.1 & 6.3 & 6.7 & 6.2 & 5.3 & 7.1 & 3.95 & 4.4 & 8.3 \\
\hline Total acidity $\left(\mathrm{g}^{-1}\right)$ & 5.09 & 5.33 & 7.57 & 6.35 & 6.97 & 7.51 & 6.64 & 6.06 & 8.39 & 5.29 & 5.13 & 10.66 \\
\hline \multicolumn{13}{|l|}{ Alcoholic } \\
\hline Alcoholic degree (\%) & 16.8 & 19.8 & 20.5 & 21.1 & 20.6 & 19.2 & 17.0 & 19.8 & 21.3 & 15.8 & 19.8 & 18.1 \\
\hline \multicolumn{13}{|l|}{ Sugars } \\
\hline Total sugars $\left(\mathrm{g}^{-1}\right)$ & 76.5 & 89.8 & 58.6 & 54.7 & 153.0 & 150.8 & - & 13.6 & 20.0 & 49.4 & 73.6 & 65.7 \\
\hline Reducing sugars $\left(\mathrm{g}^{-1}\right)$ & 32.2 & 27.8 & 20.9 & 22.8 & 33.0 & 29.6 & - & 7.3 & 11.8 & 24.8 & 34.1 & 38.0 \\
\hline Dry extract $\left(\mathrm{g}^{-1}\right)$ & 104.0 & 113.9 & 89.8 & 80.1 & 178.7 & 104.0 & 37.5 & 27.6 & 45.1 & 73.0 & 92.5 & 90.7 \\
\hline Density $\left(\mathrm{g}^{-1}, 20^{\circ} \mathrm{C}\right)$ & 1.0164 & 1.0177 & 1.0079 & 1.0033 & 1.0415 & 1.0144 & 0.9840 & 0.9845 & 0.9898 & 1.0064 & 1.0094 & 1.010 \\
\hline Baumé degree $\left(20^{\circ} \mathrm{C}\right)$ & 2.7 & 2.9 & 1.4 & 0.7 & 6.4 & 2.4 & & & & 1.1 & 1.6 & 1.9 \\
\hline \multicolumn{13}{|l|}{ Mineral } \\
\hline $\operatorname{Ash}\left(\mathrm{g} \mathrm{l}^{-1}\right)$ & 2.7 & 2.7 & 3.0 & 2.6 & 3.0 & 3.0 & 2.0 & 2.8 & 2.8 & 2.3 & 2.9 & 3.3 \\
\hline Ash alkalinity $\left(\mathrm{g}^{-1}\right)$ & 1.7 & 1.5 & 1.8 & 1.4 & 1.6 & 1.7 & 1.4 & 1.5 & 1.8 & 1.5 & 1.4 & 2.4 \\
\hline Free $\mathrm{SO}_{2}\left(\mathrm{mg} \mathrm{l}^{-1}\right)$ & 3.1 & 4.7 & 1.9 & 4.3 & 6.85 & 3.1 & 4.1 & - & 2.1 & 2.95 & 3.3 & 2.6 \\
\hline Total $\mathrm{SO}_{2}\left(\mathrm{mg} \mathrm{l}^{-1}\right)$ & 9.5 & 13.1 & 9.0 & 9.0 & 20.0 & 11.5 & 10.0 & - & 6.5 & 10.0 & 12.0 & 8.0 \\
\hline \multicolumn{13}{|l|}{ Volatiles $\left(\mathrm{mg}^{-1}\right)$} \\
\hline Acethaldehyde & 20.62 & 45.9 & 116.99 & 18.38 & 91.11 & 57.82 & $27 . .19$ & 43.82 & 85.78 & 15.60 & 37.18 & 81.48 \\
\hline Ethyl acetate & 24.01 & 53.73 & 264.36 & 30.22 & 69.98 & 101.54 & 2.98 & 32.97 & 156.24 & 26.56 & 58.87 & 125.7 \\
\hline Methanol & 51.00 & 91.41 & 88.46 & 86.57 & 70.92 & 88.44 & 57.18 & 70.79 & 152.85 & 73.95 & 73.43 & 103.4 \\
\hline Propan-1-ol & 5.10 & 5.92 & 30.94 & 14.16 & 2.69 & 16.87 & 31.65 & 22.39 & 34.08 & 7.76 & 6.33 & 21.39 \\
\hline 2-Methyl-propan-1-ol & 7.75 & 19.69 & 70.29 & 26.95 & 4.96 & 34.41 & 60.96 & 74.29 & 89.29 & 14.44 & 19.16 & 53.44 \\
\hline Butan-1-ol & 0.66 & 0.634 & 2.18 & - & 0.95 & 0.902 & 0.755 & 0.757 & 1.69 & - & 0.564 & 0.94 \\
\hline Isoamyl alcohols & 25.91 & 72.11 & 190.19 & 55.61 & 13.39 & 96.86 & 166.01 & 241.06 & 247.85 & 36.69 & 73.88 & 145.3 \\
\hline Total higher alcohols & 39.42 & 98.35 & 293.60 & 96.72 & 21.99 & 149.04 & 259.38 & 338.50 & 372.91 & 58.89 & 99.93 & 221.07 \\
\hline
\end{tabular}

a Time of storage (years).

compounds during long wine maturation. Important constituents of the aromatic profile, such as methanol from the pectin's hydrolysis, acethaldehyde from the enzymatic decarboxylation of pyruvic acid and from ethanol reduction by action of alcohol dehydrogenase and ethyl acetate from the yeast and acetic bacteria metabolism, were shown to increase with ageing.

The major changes in the aroma volatiles of Madeira wines were the great decrease in the amounts of fatty acids ethyl esters $\left(\mathrm{C}_{6}-\mathrm{C}_{16}\right)$ and acetates contrarily to the high increase of the ethyl esters of diprotic acids, such as ethyl lactate and diethyl succinate. Changes in aroma compounds during the period studied and their dependence on ageing time are shown from Figs. 3-7.

\subsubsection{Higher alcohols}

The higher alcohols are produced during yeast fermentation from two different sources: from carbohydrates by the EMP pathway and from amino acids via the Ehrlich pathway [9]. Besides higher alcohols do not change very much with ageing, some increases could even be observed, perhaps as a result of hydrolysis of the esters or evaporation during wood maturation. Major and minor higher alcohols account for 55.9-3.5\% of aroma compounds, revealing an important contribution to the flavour of wines. The increase content of 3-methylbutan-1-ol and 2-phenylethanol with time of storage (Fig. 2) can be attributed to the oxidative deamination of the free amino acids precursors, leucine and phenylalanine, respectively, by the Ehrlich mechanism, as exemplified in Fig. 3 for 3-methylbutan-1-ol. The mean content of higher alcohols considering the four studied varieties increase significantly during ageing, $24.7 \pm 6.3 \%$ in the first 11 years of conservation, due primarily, the high increase of 2+3-methylbutan-l-ol and 2-phenylethanol. The evolution of $\mathrm{C}_{6}$ alcohols, that exhibit an herbaceous and vegetative odour, is small. Fig. 2 shows the behaviour of higher alcohols during ageing for Boal wines variety with 1,11 and 25 years old. The higher alcohols are believed to contribute more to the intensity of the odour of the wine than to its quality [9].

\subsubsection{Ethyl esters}

The ethyl esters of the medium-chain fatty acids $\left(\mathrm{C}_{6}-\mathrm{C}_{12}\right)$ are produced during yeast fermentation by the reactions of ethanol and acyl-coenzyme A derivatives [10]. Their concentration is dependent on several factors mainly: yeast strain, fermentation temperature, degree of aeration and sugar contents. These compounds make a positive contribution to the general quality of wine. In young Madeira wines the ethyl esters of $\mathrm{C}_{6}$ and 


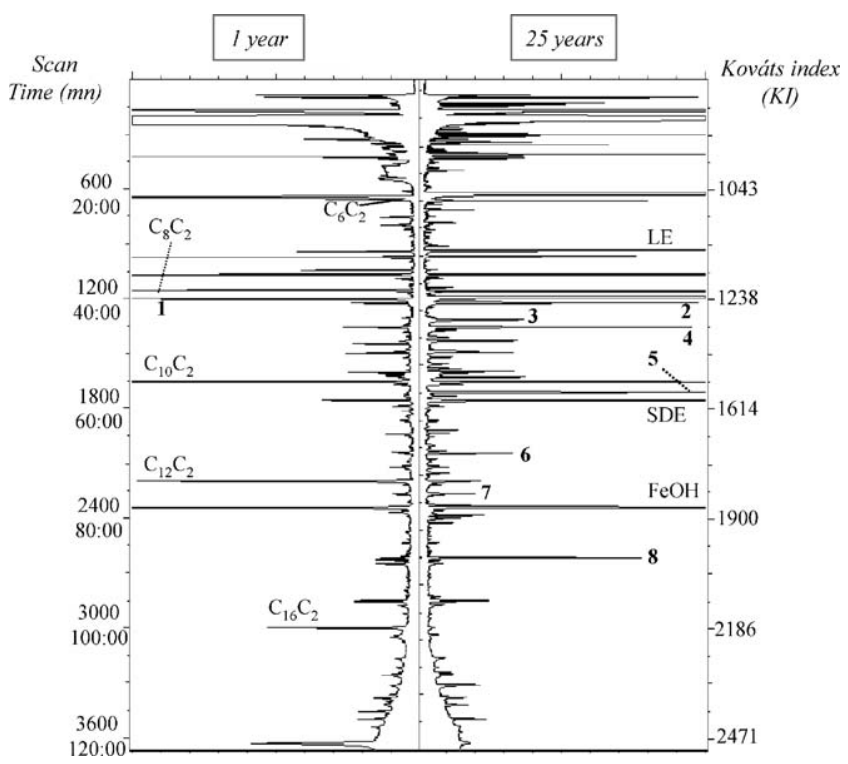

Fig. 1. GC/MS chromatograms of a dichloromethane extract from a two samples of Malvazia wines with 1 and 25 years old. Legend - (1) acetic acid; (2) 2-furfural; (3) 1,1-diethaxyethane; (4) benzaldehyde; (5) ethyl benzoate; (6) ethyl benzeneacetate; (7) benzyl alcohol; (8) ethyl 3-hydroxyhexanoate; LE: ethyl lactate; SDE: diethyl succinate; $\mathrm{FeOH}$ : 2-phenylethanol; $\mathrm{C}_{6} \mathrm{C}_{2}$ : ethyl hexanoate; $\mathrm{C}_{8} \mathrm{C}_{2}$ : ethyl octanoate; $\mathrm{C}_{10} \mathrm{C}_{2}$ : ethyl decanoate; $\mathrm{C}_{12} \mathrm{C}_{2}$ : ethyl dodecanoate; $\mathrm{C}_{16} \mathrm{C}_{2}$ : ethyl hexanoate.

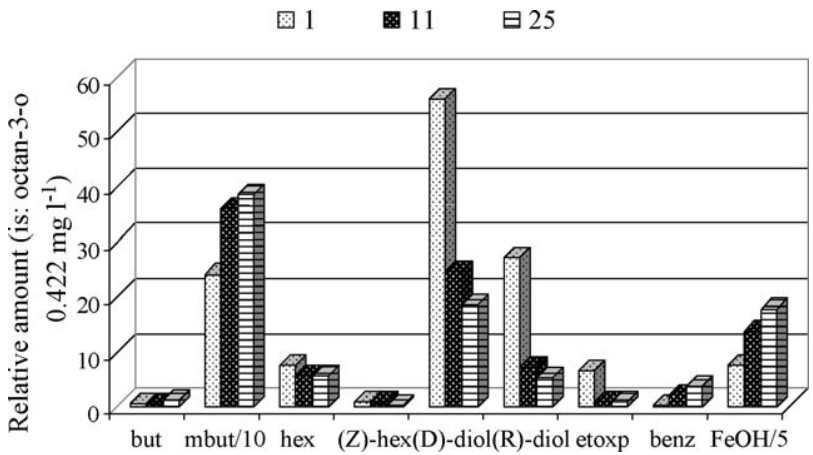

Fig. 2. Evolution of higher alcohols during ageing for Boal wines with 1, 11 and 25 years old. Legend - but: butan-1-ol; mbut: 2+3-methylbutan-1-ol; hex: hexan-1-ol; (Z)-hex: (Z)-hex-3-en-1-ol; (D)-diol: (D, L)-2,3-butanediol; $(R)$-diol: $(R, S)$-2,3-butanediol; etoxp: 3-ethoxypropan-1-ol; benz: benzyl alcohol; FeOH: 2-phenylethanol.

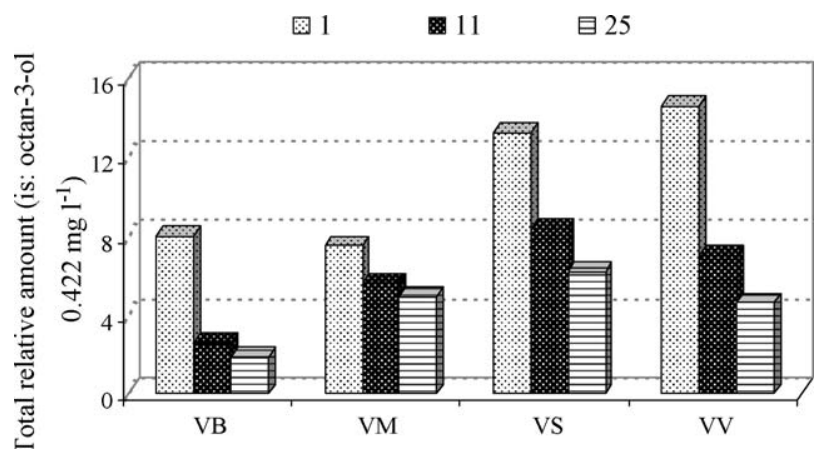

Fig. 4. Evolution profile from fatty acids ethyl esters during conservation (VB: Boal wine; VM: Malvazia wine; VS: Sercial wine; VV: Verdelho wine).

$\mathrm{C}_{8}$ fatty acids, are responsible for the "fruity" and "wine-like" aroma. Ethyl hexanoate are present at concentrations exceeding their flavour threshold. The ester composition changes due to shifts toward chemical equilibrium. These changes are influenced by wine $\mathrm{pH}$ and storage temperature. Fig. 4 compares the content of the major ethyl esters in the different wines. One of the main observations made during the ageing of Madeira wines is the great decrease in the fatty acids ethyl esters contents. This decrease is greater in the first 11 years of conservation $(45.5 \pm 6.7 \%)$ than the second 14 years of ageing $(25.1 \pm 9.3 \%)$.

Similarly, the concentration of higher alcohol acetates, with fruity scents, also decrease during ageing, contributing to the observed low fruity character of Madeira aged wines. These compounds are produced by enzymatic reactions in excess of their equilibrium concentrations. They gradually hydrolyse during storage until the approach equilibrium with their corresponding acids and alcohols is reached. The decrease showed by fatty acids ethyl esters and acetates as storage time increased could be responsible for the loss of freshness and fruitiness especially of white wines. In general, the most important differences in ethyl ester concentrations were observed during the beginning of conservation.

Contrarily, the ethyl esters of diprotic acids increase significantly with time of storage, with particular advantage to monoethyl and diethyl succinate and ethyl lactate, due the chemical esterification during the course of ageing. The linear correlation between esters from succinic acid (monoethyl and diethyl

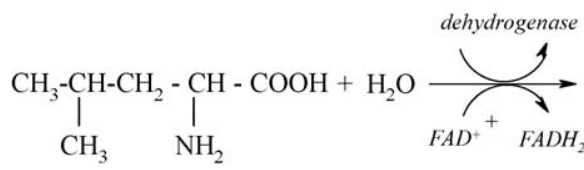

Leucine<smiles>CC(C)CC(=N)C(=O)O</smiles><smiles>CC(C)CC(C)C(C)C(=O)OC(=O)C(=O)O</smiles>

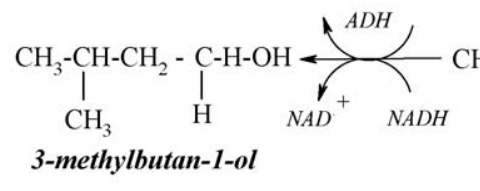

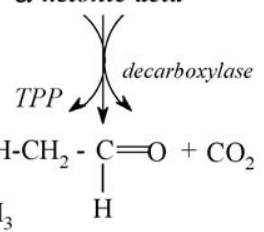

3-methyl butanal

Fig. 3. Biosynthetic pathway of 3-methylbutan-1-ol from leucine by the Ehrlich mechanism. 


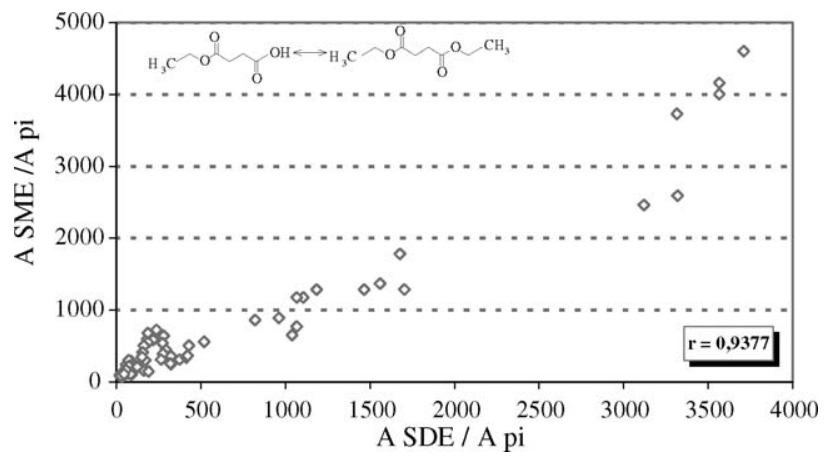

Fig. 5. Correlation between SME (ethyl succinate) and SDE (diethyl succinate) during ageing of Madeira wines $(n=86)$.

succinate) during conservation shows the equilibrium between these two compounds (Fig. 5).

\subsubsection{Fatty acids}

The formation of volatile organic acids during yeast fermentation is quantitatively slight, but it cannot be neglected from the flavour viewpoint. Anabolic and catabolic biogenetic pathways come into question for the occurrence of fatty acids in wines. Possibly, both the reaction mechanisms, that is, degradation owing to the $\beta$-oxidation in the initial phase of fermentation, as well as the anabolic processes, play a role in the formation of these compounds.

Fig. 6 shows a differentiated behaviour of fatty acid during maturation. Was observed a significant decrease in medium- and long-chain fatty acids, namely hexanoic (hex) and octanoic (oct) acids, during conservation, except for the decanoic (deca) acid whose concentration changed slightly when comparing between wines with 11 and 25 years old. Short-chain fatty acids, particularly butanoic (but) and isobutanoic (mbut) acids, increased their contents during wine ageing. As can be seen in Fig. 6 the levels of propanoic (pro) and propanedioic (malon) acids changed slightly during conservation. The hydroxybenzenacetic (hbace), hydroxybenzenpropanoic (hbp), benzoic (benz) and furoic (fur) acids, shows a great increase with time of storage.

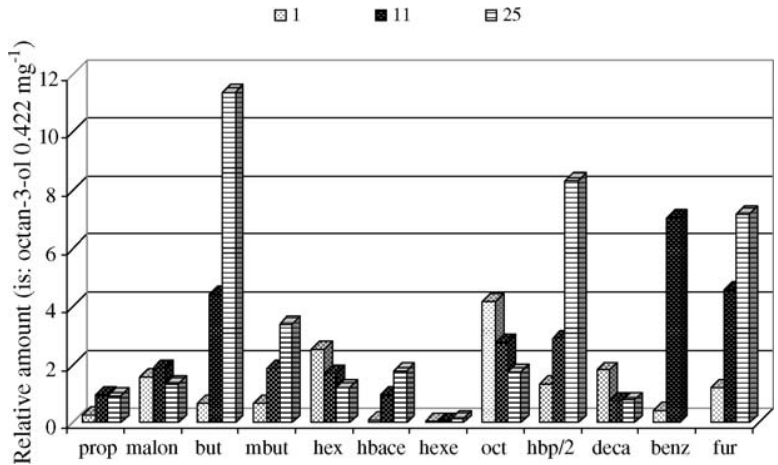

Fig. 6. Evolution of medium- and long-chain fatty acids determined in Verdelho wine samples with 1, 11 and 25 years old. Legend - prop: propanoic acid; malon: propanedioic acid; but: butanoic acid; mbut: 3-methylbutanoic acid; hex: hexanoic acid; hbace: hydroxybenzenacetic acid; hexe: (E) hex-3-enoic acid; oct: octanoic acid; hbp: hydroxybenzenpropanoic acid; deca: decanoic acid; benz: benzoic acid; fur: furoic acid.

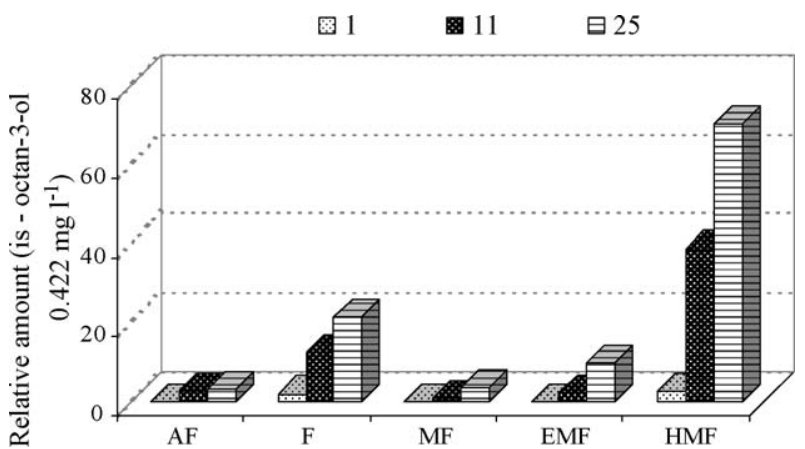

Fig. 7. Average values from furan derivatives in Malvazia wines with 1, 11 and 25 years of storage. Legend -AF: 2-acethylfuran; F: 2-furfural; MF: 5-methyl-2furfural; EMF: 5-ethoxymethyl-2-furfural; HMF: 5-hydroxymehyl-2-furfural.

\subsubsection{Furanic derivatives}

Another group of aroma compound that have been studied were the furanic compounds. The major components in this group were 2-furfural (F), 5-methyl-2-furufral (MF) and 5-hydroxymethyl-2-furfural (HMF). Minor quantities of 2-acetylfuran (AF), 5-etoxymethyl-2-furfural (EMF), 5acetoxymethyl-2-furfural (AMF) and ethyl 3-furoate were also found in present studies. 2-Furfural, 5-methyl-2-furfural, 5-hydroxymethyl-2-furfural and 5-etoxymethyl-2-furfural are formed by degradation of carbohydrates during toasting of the barrel, and may also be of sensory importance. 2-Furfural is produced when pentoses (xylose) are heated and 5-methyl-2furfural arises from the rhamnose. The quantity of furfural and 5-methyl-2-furfural in the wine depends, as well as on the age of the barrel, on the degree of wood toasting.

The minimum, maximum and medium values determined for furanic derivatives in Madeira wines, are shown in Table 2. The result shows that the higher levels wine sugars have a higher content of 5-hydroxymethyl-2-furfural. Contrary the dry wines present the lower levels of this compound that indicate furanic derivative compounds arising from sugars degradation. 2-Furfural, 5-methyl-2-furfural and 5-hydroxymethyl2-furfural showed a similar evolution in the four wines up to 25 years of ageing; afterwards the accumulation of these compounds was much higher in the Malvazia and Boal wines, with higher alcoholic degree (19.2\%, v/v, and $17.8 \%$, v/v, respectively) than in the Verdelho $(16.9 \%$, v/v) and Sercial $(16.8 \%, \mathrm{v} / \mathrm{v})$ wines. In the time considered (1-25 years old), was observed a significantly increment of contents of these compounds, with distinction for 2-furfural and 5-hydroxymethyl2-furfural (Fig. 7). The correlation coefficients calculated for 2-furfural, 5-methyl-2-furfural and 5-hydroxymethyl-2-furfural (HMF), 0.921, 0.906 and 0.916, respectively, demonstrated linear behaviour of these compounds over time during ageing, which makes these compounds, wine age indicators. The same behaviour was observed by Silva Ferreira et al. [4] for Porto aged wines.

At anaerobic conditions and at wine $\mathrm{pH}$ value, the 5hydroxymethyl-2-furfural given rise the 5-ethoxymethyl-2furfural [11]. This compound can also be formed by condensation between ethanol and 5-hydroxymethyl-2-furfural [12]. Depending at the concentration, the 5-ethoxymethyl-2-furfural 
Table 2

Relative amounts (internal standard: octan-3-ol, $0.422 \mathrm{mg} \mathrm{l}^{-1}$ ) of some volatile compounds in young (1 year) and old (25 years) Madeira wines

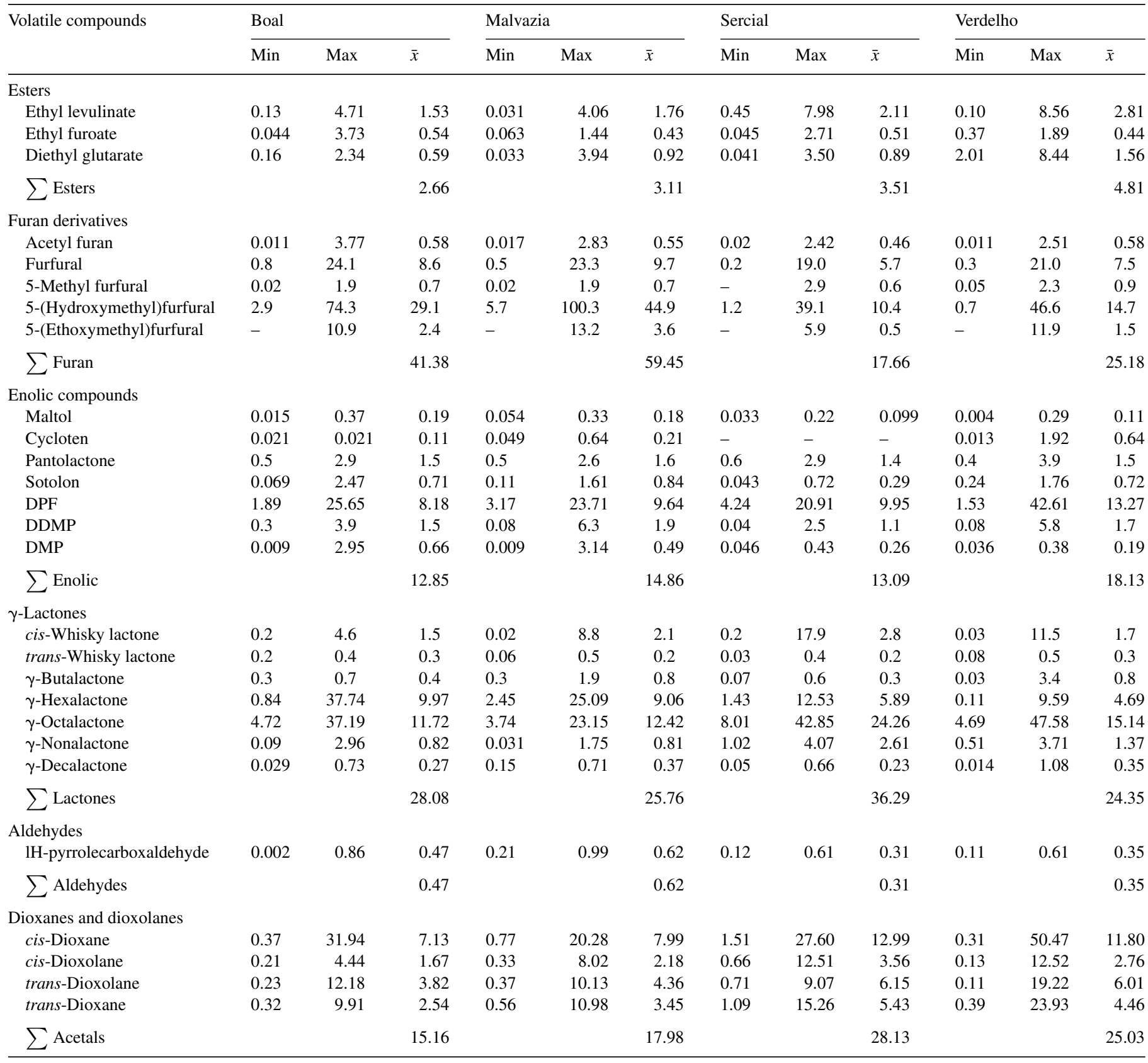

Min: minimum value; Max: maximum value; $\bar{x}$ : medium value.

is responsible for several descriptors like spice and curry notes. The identification by GC/MS (Fig. 8e), present a fit of $84.2 \%$ with the proposed by NIST fragmentation. The retention index in the Stabilwax capillary column is 2128 .

The behaviour observed for 5-ethoxymethyl-2-furfural in course of ageing process is similar to the other furanic derivatives, with apparent linear increment with time $(r=0.912)$ of wine conservation.

Another compound with a great impact on aged Madeira wines flavour is 3-hydroxy-4,5-dimethyl-2 $(5 \mathrm{H})$-furanone (sotolon). Considering the categories, it was observed that sotolon concentration was clearly dependent on the time of barrel storage, as the high correlation coefficient $(r=0.917)$ demonstrated. These trends are in agreement with the previously reported behaviour for Port wines [13]. The concentrations of sotolon increase with time from $100 \mu \mathrm{gl}^{-1}$ in wines with 6 years old to about $1000 \mu \mathrm{g} 1^{-1}$ in wines with 25 years old. The highest contents were observed for wines with 25 years old, higher than $1 \mathrm{mgl}^{-1}$. If we consider the wines from each variety, the linear correlation is very pronounced.

The results show that higher levels of residual sugars content correspond to a higher content of 5-hydroxymethyl-2furfural. On the contrary the "dry" wines present lower levels of this compound indicating the formation of furanic 

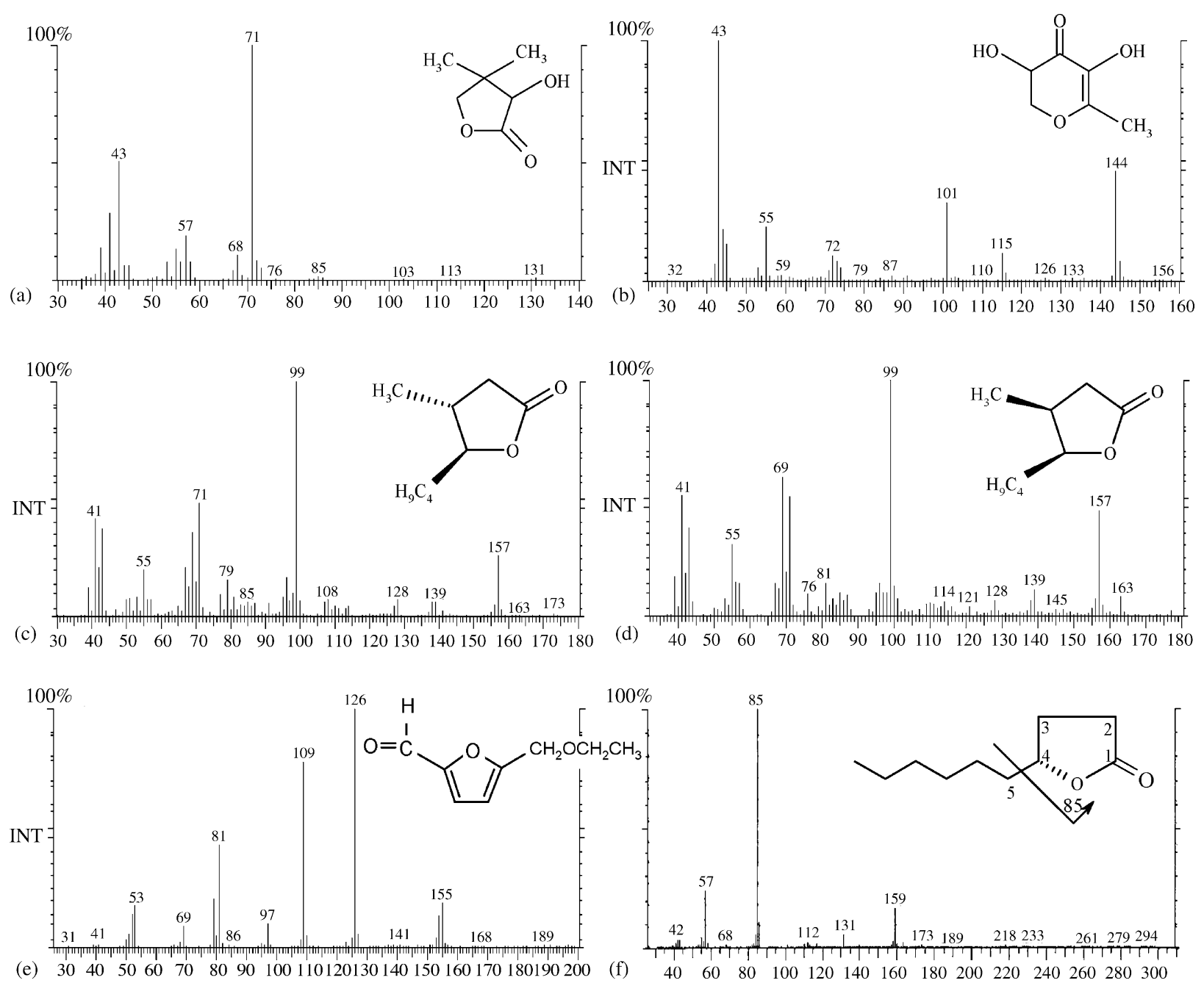

Fig. 8. Mass spectra (GC/MS, El $70 \mathrm{eV}$ ) and chemical structure of some compounds responsible for the "toasty" and "caramel-like" odours: (a) pantolactone; (b) DDMP; (c) trans-whisky lactone; (d) cis-whisky lactone; (e) 5-ethoxy-methyl-2-furfural; (f) $\gamma$-decalactone, showing origin of the base peak $m / z=85$ in the mass spectrum.

derivatives compounds arising from degradation of sugars (Table 2).

\subsubsection{Enolic compounds}

Oak wood used in barrels for ageing wine produces a great number of volatile and odoriferous compounds. Were identified already known substances, arising from Maillard reactions, such as: 2-hydroxy-3-methyl-2-cyclopenten-1-one (cyclotene) formed from the less basic Amadori intermediates [14]; 3-hydroxy2-methyl-4H-pyran-4-one (maltol) from thermal degradation of substituted glucose-4- $O$ structures [15]; 2,3-dihydro-3,5dihydroxy-6-methyl-4H-pyran-4-one (DDMP); 4,5-dihydro-3hydroxy-4,4-dimethyl-2(3H)-furanone (pantolactone) formed during alcoholic fermentation due to the action of a reductase [16]; 3,5-dihydroxy-2-methyl-4H-pyran-4-one (DMP) byproduct of heating maltose in the presence of glycine; as compounds which contribute to the "toasty" and "caramel" aroma. Fig. 8 shows the mass spectra and the corresponding chemical structure of some enolic compounds.

Among the compounds released from the wood, the oak lactones (cis- and trans- $\beta$-methyl- $\gamma$-octalactones) are the most important from o sensory point of view. They arise from lipid oxidation and are known to increase with time of storage. No significant differences were found among the enolic compounds considering the wine varieties studied. Table 2 shows the obtained values for 1,11 and 25 years.

\subsection{6. $\gamma$-Lactones}

$\gamma$-Lactones are among the most important to the sensory characteristics of wines aged in oak wood. These compounds are formed by cyclisation of the corresponding $\gamma$-hydroxycarboxylic acids. The odour of these lactones are described as being "fruity" and in some cases as "coconut-like, fruity" ( $\gamma$-hexalactone); "coconut-like" $(\gamma$-octalactone $)$; "peach-like, milky" ( $\gamma$-decalactone); "fruity, sweet floral" ( $\gamma$-dodecalactone). Due to their distinct fruity flavour, the basic biosynthetic mechanisms should be of great interest. According to Haffner and Tressl [17], $\gamma$-lactones arising from biotransformation of oleic acid, as represented in scheme of Fig. 9. The same authors postulate that the first enzymatic step should be the $(R)$-12-enantioselective hydroxylation of 


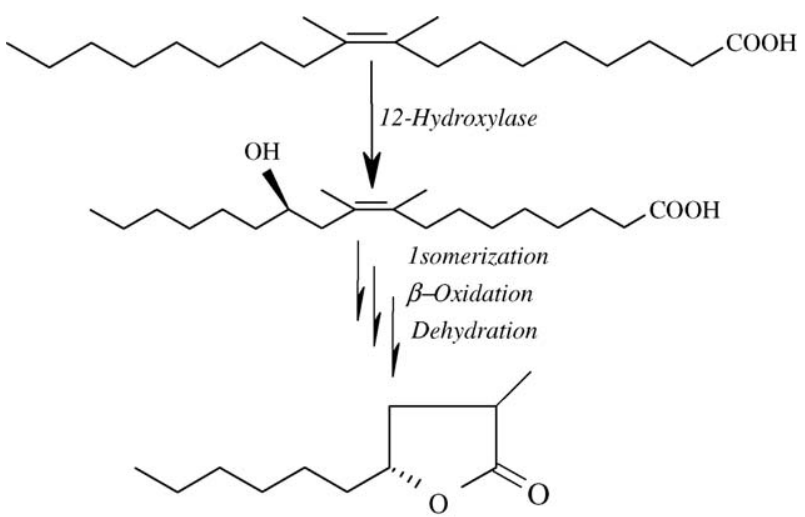

Fig. 9. Proposed transformation of oleic acid to $\gamma$-decalactone [17].

oleic acid, catalysed by a hydroxylase, followed by the four $\beta$-oxidation cycles and dehydration.

Under standard electron impact GC/MS conditions $(70 \mathrm{eV})$, loss of the alkyl substituent at $\mathrm{C}_{4}$ is favoured, leading to the fragment ion $m / z=85$ (tetrahydropyranonyl cation) the most important in the mass spectral analysis of $\gamma$-lactones with saturated ring moieties. The characteristic mass spectra of $\gamma$-octalactone, is showed in Fig. 8f. The obtained results show that these compounds increase greatly with time of storage.

\subsubsection{Dioxanes and dioxolanes}

The heterocyclic acetals: cis- and trans-5-hydroxy-2methyl-1,3-dioxane (cis- and trans-dioxane) and cis- and trans-4-hydroxymethyl-2-methyl-1,3-dioxolane (cis- and transdioxolane), are formed by acetalisation between acetaldehyde and glycerol, one of the major wine components. This reaction is highly favoured at wine $\mathrm{pH}$ and by the high content of the acetaldehyde. These compounds were identified in different types of wines $[18,19]$ and their evolution in Port wines was described in detail [18].

As shown in Fig. 10 for the cis-dioxane and cis-dioxolane, the relative amounts of the acetals increase with time and show high linear correlation with age (correlation coefficient $>0.9$ ). This linear correlation with the wine age allows the easy differentiation of young and old Madeira wines and can be used as an indicator of Madeira wine age.

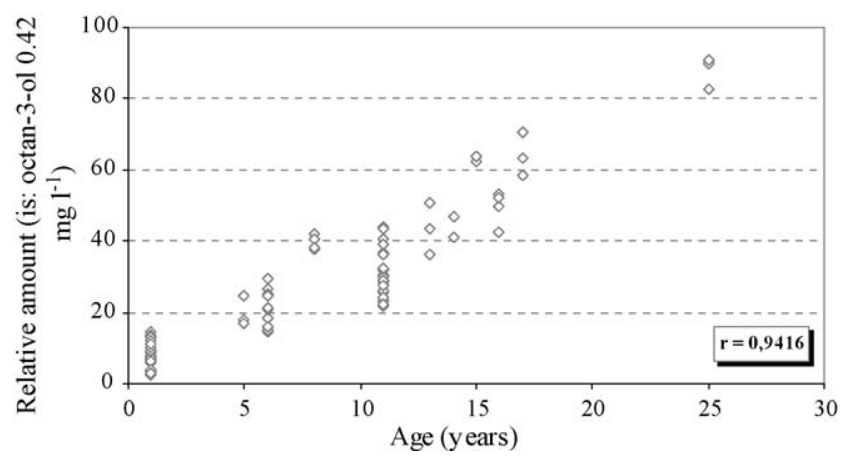

Fig. 10. Evolution of acetals (dioxanes and dioxolanes) in fortified Madeira wines during ageing $(n=86)$.

\section{Conclusions}

The aroma compositions of Madeira wines with 1, 11 and 25 years old were analysed. A significant variation of volatile compounds during wine ageing was observed. The results show a great decrease from fatty acids ethyl esters $\left(\mathrm{C}_{6}-\mathrm{C}_{16}\right)$ as the storage time gets longer, contrary to the high increase from ethyl esters of diprotic acids, such as ethyl lactate and diethyl succinate. This variation does not occur at the same rate during ageing but, generally, seems to be faster at the beginning of the ageing period.

The variation of average content of higher alcohols is uncharacteristic and small during ageing.

The results show that there is a strong correlation between sotolon, sugar derivatives: 2-furfural, 5-methyl-2-furfural, 5hydroxymethyl-2-furfural and 5-ethoxymethyl-2-furfural, with wine ageing. The furanic derivatives present in aged wines do not originate exclusively from the oak cask but can also be formed from the carbohydrates occurring in wines during the ageing period.

It was observed that during ageing, the concentration of sotolon increased with time in a linear fashion $(r=0.917)$. The highest concentration of sotolon was found in wines with the highest residual sugar contents, considering the same time of storage. These findings indicate that the kinetic of sotolon formation is closely related with residual sugar contents suggesting that this molecule may come from a component like sugar.

Concerning to the heterocyclic acetals, the results shows a linear increase of dioxane and dioxolane isomers with time. The ratio between cis- and trans-forms remains constant independently of the wine age $2.35 \pm 0.31$ for cis-/trans-dioxane and $1.60 \pm 0.18$ for cis-/trans-dioxolane.

\section{Acknowledgments}

We gratefully acknowledge the FEDER through INTERRREG III project the financial support for this investigation and Instituto do Vinho da Madeira and Madeira Wine Company for the supply of wine samples.

\section{References}

[1] J.S. Câmara, P. Herbert, J.C. Marques, M.A. Alves, Anal. Chim. Acta 513 (2004) 203.

[2] I. Cutzach, P. Chatonnet, D. Dubourdieu, J. Int. Sci. Vigne Vin 32 (1998) 99.

[3] C. Cantareli, 2nd Symposium International d'Oenologie, BoredeauxCognac, 1967, p. 391.

[4] A.C. Silva Ferreira, J.C. Barbe, A. Bertrand, J. Agric. Food Chem. 51 (2003) 4356.

[5] A.C. Silva Ferreira, J.C. Barbe, A. Bertrand, J. Agric. Food Chem. 50 (2002) 2560.

[6] J.S. Câmara, P. Herbert, J.C. Marques, M.A. Alves, A.C. Silva Ferreira, Anal. Bioanal. Chem. 373 (2003) 1221

[7] M. Forina, C. Armanino, M. Castino, M. Ubigli, Vitis 25 (1986) 189.

[8] P. Munch, P. Schieberle, Agric. Food Chem. 46 (1998) 4695.

[9] P. Etiévant, Wine, in: H. Maarse (Ed.), Volatile Compounds in Foods and Beverages, TNO-CIVO Food Analysis Institute, Zeist, The Netherlands, 1991, pp. 486-546. 
[10] C. Nordest, A. Bergtsson, P. Bennet, I. Lindström, Proceedings of the 15th Congress of the European Brewery Convention, Elsevier Scientific Publishing Co., Nice, Amsterdam, 1975, p. 581.

[11] I. Cutzach, P. Chatonnet, R. Henry, D. Dubourdieu, J. Agric. Food Chem. 47 (1999) 1663.

[12] J.S. Câmara, J.C. Marques, M.A. Alves, A.S. Silva Ferreira, J. Agric. Food Chem. 52 (2004) 6765.

[13] A.C. Silva Ferreira, T. Hogg, P. Guedes de Pinho, J. Agric. Food Chem. $51(2003) 1373$.
[14] F.D. Milss, J.E. Hodge, Carbohydr. Res. 51 (1976) 9.

[15] J.E. Hodge, B.E. Fisher, E.C. Nelson, Am. Soc. Brewing Chemist Proc. (1963) 84.

[16] H. King, D.R. Wilken, J. Biol. Chem. 247 (1972) 4096.

[17] T. Haffner, R. Tressl, J. Agric. Food Chem. 44 (1996) 1218.

[18] A.C. Silva Ferreira, Ph.D. Thesis (no. 59.3), Université Victor Segalen Bordeaux 2, 1998.

[19] R.F. Simpson, J. Sci. Food Agric. 31 (1980) 214. 